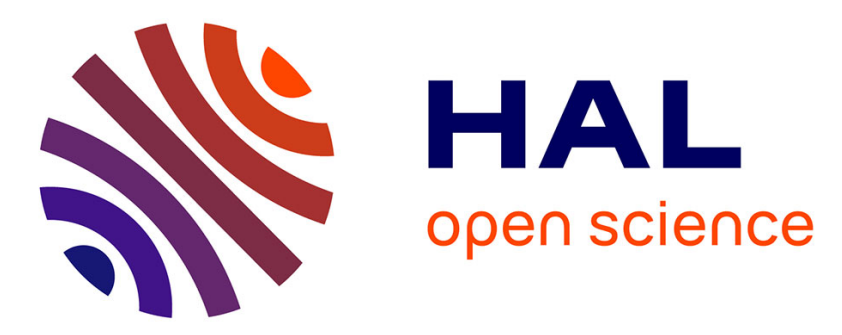

\title{
Method to compensate dispersion effect applied to time domain reflectometry
}

\author{
L. Sommervogel, L. El Sahmarany, L. Incarbone
}

\section{To cite this version:}

L. Sommervogel, L. El Sahmarany, L. Incarbone. Method to compensate dispersion effect applied to time domain reflectometry. Electronics Letters, 2013, 49, pp.1154 - 1155. 10.1049/el.2013.1042 . cea-01777448

\section{HAL Id: cea-01777448 https://hal-cea.archives-ouvertes.fr/cea-01777448}

Submitted on 24 Apr 2018

HAL is a multi-disciplinary open access archive for the deposit and dissemination of scientific research documents, whether they are published or not. The documents may come from teaching and research institutions in France or abroad, or from public or private research centers.
L'archive ouverte pluridisciplinaire HAL, est destinée au dépôt et à la diffusion de documents scientifiques de niveau recherche, publiés ou non, émanant des établissements d'enseignement et de recherche français ou étrangers, des laboratoires publics ou privés. 


\section{Method to compensate dispersion effect applied to time domain reflectometry}

\section{Sommervogel, L. El Sahmarany and L. Incarbone}

A new post-processing approach which aims at reducing the dispersion effect of the wave travelling inside a waveguide is presented. The main objective is to improve the time domain reflectometry measurements performed over long-distance cables, relative to the wavelength $\lambda$. A real-case scenario is presented so that the gain of this method can be appreciated.

Introduction: Cables are present in almost all modern systems. These cables are subject to several electrical, chemical and mechanical stresses. Depending on the application area, such degradation can lead to hard faults (short or open circuits) and therefore catastrophic consequences [1]. Reflectometry methods are commonly used for assessing transmission lines [2]. This Letter overcomes reflectometry's limitations by proposing a signal processing technique based on dynamic crosscorrelation for the detection and the localisation of faults over longdistance cables.

Reflectometry basics: Time-domain reflectometry reduced to its simplest form consists in sending a high-frequency wave down a cable and waiting for the probe signal to be reflected back to the source from any singularity it may have encountered during its propagation. This operation can be modelled as a linear filter, described by its impulse response $h$, which transforms the probe signal $x$ into a reflected signal $y$.

This can be written in the time domain as

$$
y(t)=h(t) * x(t)
$$

where $*$ denotes the convolution operator. If the cable were ideal, it would only introduce a delay and $h$ would be sparse with only one nonzero point, at the end of the cable

$$
h(t)=\delta(t-\tau)
$$

where $\delta$ denotes the Dirac function and $\tau$ is the delay required for the signal to reach the cable's end and come back. From (1) and (2), we have immediately

$$
y(t)=x(t-\tau)
$$

The probe signal $x$ can be a pulse, but this kind of waveform is not always suitable, depending on the application requirements. A pulseshaped signal is a bad choice if part of the spectrum must be preserved to host data communication for instance. That is the reason why new techniques and therefore new shapes have been brought to light; for instance, modulated binary random sequences, chirps or multi-carrier signals [3], which behave as pseudo-noises over a wideband spectrum. However, they require a cross-correlation post-processing to be workable and easily understood by a human operator.

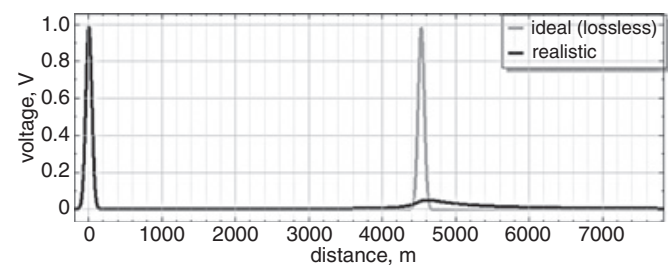

Fig. 1 Realistic simulation (black) against ideal case (grey)

From another point of view, when the length of the cable under test is very important compared with the wavelength $(l-100 \lambda)$, two very annoying phenomena begin to take place: first, attenuation not due to the losses in the cable, but also the dispersion effect, which happens when all the spectral components of the probe signal do not travel at the same speed inside a transmission line. This leads to a critical modification of the input signal shape: the injected pulse can be reflected as a flattened bump (see Fig. 1), which makes the reference points required to estimate the delay $\tau$ difficult to determine. To understand precisely, let us see what happens in the frequency domain, where the Fourier transform of (2) would be expressed as

$$
H(\omega)=\exp (-2 \mathrm{j} \omega \tau)
$$

Besides, it is well known that another more common expression for $H$ can be obtained from the telegrapher's [4] equations

$$
H^{\prime}(\omega)=\exp \left[\begin{array}{lll}
-2 & \gamma(\omega) & l
\end{array}\right]
$$

where $\gamma$ is the propagation constant and can be expressed as a function of the transmission line's $R L C G$ distributed parameters [5]

$$
\gamma(\omega)=\sqrt{(R+\mathrm{j} L \omega)(G+\mathrm{j} C \omega)}
$$

From (4) and (5), we have

$$
\gamma(\omega)=\mathrm{j} \omega \tau / l=\mathrm{j} \omega / v
$$

$\gamma$ in (7) is purely imaginary and this can be achieved only if the transmission line is lossless, which is never the case in real life. Moreover, if $R, L, C$ and $G$ depend on the frequency, along with $v$, which leads to the dispersion effect we would like to account for.

Principles of operation: Since we will work here with a method based on cross-correlation, the main advantage is that it can be applied to any probe signal type and waveform, as long as its autocorrelation is close to being peak-shaped. Indeed, correlation works also with pulse-shaped signals: it dramatically reduces white noise and smoothes the response. Simple cross-correlation expresses as follows:

$$
R_{x y}(t)=\int_{-\infty}^{+\infty} x(\theta) y(t+\theta) \mathrm{d} \theta
$$

This operation measures the similarity of the reflected waveform and the reference as a function of a time-lag applied between them. Currently, the post-processing methods used in reflectometry do not take the propagation medium into account. An assumption is made about the velocity $v$, so that the time measurements can be converted into distances. This also means that in (8), the reference $x$ never changes during the calculation. The whole idea of our technique is to modify the reference according to the time-lag

$$
R_{x y}^{\prime}(t)=\int_{-\infty}^{+\infty} x_{\theta}(\theta) y(t+\theta) \mathrm{d} \theta
$$

Since the reference is now changing, our method will be qualified as dynamic correlation. This makes sense only if the cable can be modelled (even though it is not perfectly precise), so that the dispersion effect can be estimated. To do so and find out how to calculate $x_{\theta}$, we need to work in the frequency domain. Let $H_{\theta}$ be the impulse response we would have had if the length of the transmission line was $v\left(\omega_{0}\right) \theta / 2$, where $\omega_{0}$ denotes the working frequency

$$
H_{\theta}(\omega)=\exp \left[-\gamma(\omega) v\left(\omega_{0}\right) \theta\right]
$$

Thus, we can express the reference signal which would have travelled during the time span $\theta$ as $X_{0}(\omega) H_{\theta}(\omega)$, where $X_{0}(\omega)=X(\omega)$. However, this would introduce a time offset and the result of (9) would be incorrect. That is the reason why a phase delay term should be added to the final expression

$$
X_{\theta}(\omega)=X_{0}(\omega) \exp \left[-\gamma(\omega) v\left(\omega_{0}\right) \theta\right] \exp (+\mathrm{j} \omega \theta)
$$

From (7) and (11), we show that if the cable is lossless and LC is constant, $\forall \theta X_{\theta}=X_{0}=X$. The dynamic correlation thus behaves as a simple one. Finally, we can compute the result in the time domain

$$
x_{\theta}(t)=\frac{1}{A_{\theta}} \int_{-\infty}^{+\infty} X_{\theta}(\omega) \exp (\mathrm{j} \omega t) \mathrm{d} \omega
$$

where $A_{\theta}$ is for normalisation. Once $R_{x y}^{\prime}(t)$ has been calculated, $\tau$ can be easily deduced by locating the two extrema of the resulting curve.

Simulation results: The efficiency of the new approach is first evaluated with a simulation tool. Fig. 1 sheds light on the problem we could have with a $4500 \mathrm{~m}$ twisted pair.

We can see from this simulation that the reflected signal becomes very weak over a long distance. Moreover, locating the extrema of the curve to determine the round trip delay $\tau$ would clearly be inaccurate (this would lead to an error of approximately $100 \mathrm{~m}$ ). On the other hand, 
Fig. 2 shows the result of dynamic correlation, so that we can see the accuracy improvement and the efficiency of this method.

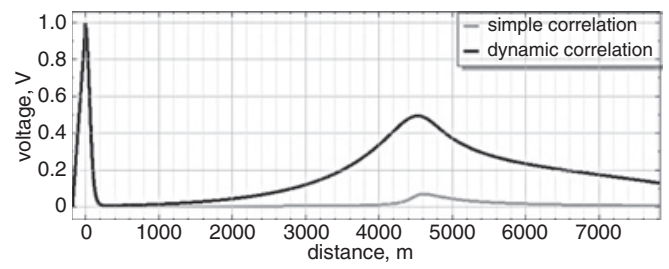

Fig. 2 Realistic simulation post-processed with simple correlation (grey) against dynamic correlation (black)

If we look at the location of the curve extrema, we find $4580 \mathrm{~m}$ for the simple correlation and $4510 \mathrm{~m}$ for the dynamic one. Therefore, the simple one practically shows no improvement (the location was $4590 \mathrm{~m}$ without processing), whereas our technique is very close to the correct value of $4500 \mathrm{~m}$ (relative error is $0.25 \%$ ). Moreover, the amplitude is greater with dynamic correlation.

Now, let us simulate what happens if there are two defects on the same cable: the open circuit's location is still at $4500 \mathrm{~m}$, but we also add a $50 \Omega$ serial resistance located at $2000 \mathrm{~m}$, as illustrated in Fig. 3.

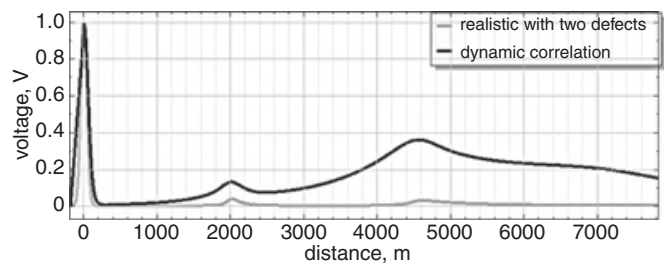

Fig. 3 Realistic simulation with two defects (grey) against post-processed with dynamic correlation (black)

The serial resistance induces a mismatch reflection whose amplitude is quite the same as the open end. We note that our method successfully enables the detection and localisation of both defects at 1995 and $4520 \mathrm{~m}$, respectively.

Experimental results: The method was then applied to a real-life scenario: detecting a cut in a cable used for data transmission along a railway. A twisted pair cable inside a bundle is studied, which means the model used [6] for computation will be slightly imprecise. However, Fig. 4 clearly indicates the accuracy of localisation we gain by using dynamic correlation.

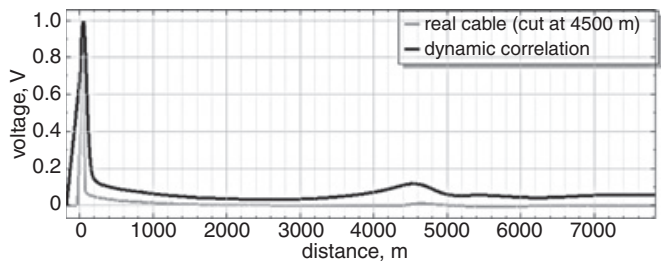

Fig. 4 Real measurement (grey) against dynamic correlation (black)

We note a slight change of shape in the waveforms due to the capacitive effect of the measurement device's output. Moreover, we realise how small the reflection at $4500 \mathrm{~m}$ is, and therefore, very hard to detect. However, dynamic correlation successfully manages to detect and locate the cut at $4520 \mathrm{~m}$.

Conclusion: This Letter presents the efficiency of a new postprocessing method applied to the TDR: the dynamic cross-correlation. It is compliant with most probe signals classically used and greatly improves the accuracy of hard faults localisation in point-to-point very long cables. Since a correlation operation is often required anyway, our method does not overload the central processing unit in case it would be implemented in an embedded device.

(C) The Institution of Engineering and Technology 2013

2 April 2013

doi: 10.1049/el.2013.1042

L. Sommervogel, L. El Sahmarany and L. Incarbone (Laboratoire de Fiabilisation des Systèmes Embarqués, CEA, LIST, Saclay NanoInnov PC172, 91191 Gif-sur-Yvette, Cedex, France)

E-mail: lola.el-sahmarany@cea.fr

\section{References}

1 Wheeler, K.R., and Timucin, D.A.: 'Aging aircraft wiring fault detection survey'. June 2007

2 Auzanneau, F.: 'Wire troubleshooting and diagnosis: review and perspectives', Prog. Electromagn. Res. B, 2013, 49, pp. 253-279

3 Lelong, A.: 'On line wire diagnosis using multicarrier time domain reflectometry for fault location', IEEE Sens., Christchurch, New Zealand, October 2009, pp. 751-754

4 Sahmarany, L., Auzanneau, F., and Bonnet, P.: 'Novel reflectometry method based on time reversal for cable aging characterization'. 58th IEEE Holm Conf., Portland, OR, USA, September 2012

5 Paul, C.R.: 'Analysis of multiconductor transmission lines' (Wiley-Interscience Publication, 1994)

6 Kien Truong, T.: 'Twisted-pair transmission-line distributed parameters', The EDN Network, 31 December 1969 